\title{
Los procesos matemáticos en la práctica docente: análisis de la fiabilidad de un cuestionario de evaluación
}

\section{Mathematical processes in teaching: analysis of the reliability of an evaluation questionnaire}

\author{
Antonio Maurandi López*1 \\ amaurandi@um.es \\ Ángel Alsina** \\ angel.alsina@udg.edu \\ Claudia Coronata Segure*** \\ ccoronata@uc.cl \\ *Universidad de Murcia, España \\ **Universitat de Girona, España \\ ***Pontificia Universidad Católica de Chile, Chile
}

\section{Resumen:}

Este artículo presenta los resultados del análisis de la fiabilidad interna de un cuestionario que ha sido construido para evaluar la presencia de los procesos matemáticos en las prácticas de enseñanzaaprendizaje de las matemáticas. Una vez validado el instrumento mediante juicio de expertos en un estudio previo, 20 expertos evaluadores administran el instrumento a 95 maestros de colegios de Educación Infantil y Primaria de la Comunidad de Murcia (España). Los datos obtenidos han sido analizados con el paquete estadístico $R$ versión 3.1.0 sobre una plataforma i686pc-linux-gnu (32-bit). El coeficiente alfa de Cronbach, basado en puntuaciones estandarizadas, muestra que la consistencia interna es buena (coeficiente alfa medio .789 en Infantil y .86 en Primaria), siendo

\begin{abstract}
:
This article presents the results of an internal reliability analysis of a questionnaire designed to evaluate the presence of mathematical processes in the practices used to teach and learn mathematics. After validating the instrument by means of expert judgement in a prior study, 20 expert evaluators administered the instrument to 95 teachers of preschool and primary education in the Community of Murcia (Spain). The data obtained was analysed with the R-3.1.0 statistical package on an i686-pc-linux-gnu (32-bit) platform. Based on standardised scores, the Cronbach alpha shows a good level of internal consistency (median alpha coefficient .789 for Preschool and .86 for Primary), with the lowest level corresponding to the process of "representation" in preschool, with a
\end{abstract}

1 Dirección para correspondencia (correspondence address):

Antonio Maurandi López. Área de Didáctica de las Ciencias Matemáticas, Universidad de Murcia, Facultad de Educación, Campus de Espinardo, s/n. 30100 Murcia (España). 
Los procesos matemáticos en la práctica docente: análisis de la fiabilidad de un cuestionario de evaluación

Antonio Maurandi López, Ángel Alsina y Claudia Coronata Segure

el valor más bajo el correspondiente al proceso "representación" en Educación Infantil, con un valor de .74, que puede valorarse también como una consistencia interna aceptable.

\section{Palabras clave:}

Procesos matemáticos; práctica docente; cuestionario de evaluación; análisis de la fiabilidad. value of .74 , which is still considered to be an acceptable level of internal consistency.

\section{Key words:}

Mathematical processes; teaching practices; evaluation questionnaire; reliability analysis.

\section{Résumé:}

Cet article présente les résultats de l'analyse de la fiabilité interne d'un questionnaire qui a été construit pour évaluer la présence de processus mathématiques dans les pratiques d'enseignement et d'apprentissage des mathématiques. L'instrument a été administré à 95 enseignants de l'éducation infantile et primaire de la Communauté de Murcie (Espagne). Les données obtenues ont été analysées avec le logiciel statistique $R$ Version 3.1.0 sur une plate-forme i686-pc -linux -gnu (32-bit). L'alpha de Cronbach, basé sur des scores normalisés, montre que la cohérence interne est bonne (coefficient alpha moyen .789 dans l'éducation infantile et .86 dans éducation primaire), la plus faible valeur correspondant au processus «représentation» dans l'éducation de la petite enfance, d'une valeur .74, qui peut également être évaluée comme une cohérence interne acceptable.

\section{Mots clés:}

Procédés mathématiques; pratique de l'enseignement; questionnaire d'évaluation; analyse de la fiabilité.

Fecha de recepción: 15-3-2016

Fecha de aceptación: 5-4-2017

\section{Introducción}

La educación matemática es una disciplina atenta a los retos sociales y procura darles respuesta. En este sentido, uno de los focos de interés contemporáneos es formar a personas que paulatinamente sean capaces de usar de manera comprensiva y eficaz los conocimientos matemáticos en las situaciones de la vida cotidiana donde estos conocimientos son necesarios, tal como señalan diversos organismos de gran repercusión internacional como el National Council of Teachers of Mathematics (NCTM, 2000) o bien la Organisation for Economic Co-operation and Development (OECD, 2004, 2007).

La investigación en educación matemática se ha ocupado en los últimos tiempos de dar respuesta a este reto desde sus diferentes ámbitos, descritos por Llinares (2008): el análisis didáctico y la organización del contenido matemático, el desarrollo profesional, la construcción del co- 
nocimiento matemático o el análisis de la práctica del profesor. Progresivamente, los resultados de estos trabajos se han ido incorporando en los currículos de matemáticas de la mayoría de países. En este sentido, una de las innovaciones curriculares que ha tenido mayor eco en el últimos tiempos es que se debería dejar de formar a personas académicamente competentes (o sólo académicamente competentes) para pasar a formar personas socialmente competentes. En otras palabras, los currículos han incorporado con fuerza la idea de "alfabetización matemática" (mathematical literacy) para referirse a las capacidades individuales de los estudiantes para analizar, razonar y comunicar eficazmente cuando formulan y resuelven problemas matemáticos en una variedad de dominios y situaciones (OECD, 2004), o de "competencia matemática" (mathematical competence), para resaltar su carácter funcional (OECD, 2007).

Con el propósito de fomentar que la sociedad tenga la capacidad de pensar y razonar matemáticamente, el NCTM plantea diez estándares que constituyen un conjunto coherente de conocimientos y competencias matemáticas: los estándares de contenidos (números y operaciones, álgebra, geometría, medida y análisis de datos y probabilidades) que describen explícitamente los contenidos que deberían aprenderse, y los estándares de procesos (resolución de problemas, razonamiento y prueba, comunicación, conexiones y representación), que ponen de relieve las formas de adquisición y uso de los contenidos (NCTM, 2000).

Varios autores (De Guzman, 2001; Niss, 2002; Alsina, 2011, 2015; entre otros) indican que los procesos matemáticos son una de las claves para conseguir este propósito:

En la situación de transformación vertiginosa de la civilización en la que nos encontramos, está claro que los procesos verdaderamente eficaces de pensamiento, que no se vuelven obsoletos con tanta rapidez, es lo más valioso que podemos enseñar a nuestros jóvenes. En nuestro mundo científico e intelectual tan rápidamente mutando vale mucho más proveerse de procesos de pensamiento útiles que de contenidos que rápidamente se convierten en ideas inertes (De Guzmán, 2001, p. 9)

Para este autor la matemática es, sobre todo, saber hacer, es una ciencia en la que el método predomina claramente sobre el contenido. En esta misma línea, Niss (2002) señala la necesidad de substituir los currículum de matemáticas orientados a la adquisición de contenidos, 
puesto que se centran exclusivamente en la adquisición de símbolos y de técnicas, por currículum orientados al uso significativo de estos contenidos en una variedad de situaciones en las cuales las matemáticas pueden ejercer un papel. Alsina $(2011,2015)$ ofrece orientaciones al profesorado para el trabajo integrado de los diferentes bloques de contenido en conexión con los procesos para favorecer la autonomía mental de los alumnos, potenciando la elaboración de hipótesis, las estrategias creativas de resolución de problemas, la discusión, el contraste, la negociación de significados, la construcción conjunta de soluciones y la búsqueda de formas para comunicar planteamientos y resultados. Se fija, pues, la atención en un enfoque social que ayude a los alumnos a gestionar sus conocimientos, sus habilidades y sus emociones para conseguir un objetivo a menudo más cercano a situaciones funcionales y en contextos de vida cotidiana que a su uso académico.

Alsina y Coronata (2014) indican que la planificación y gestión de prácticas docentes que incorporen los procesos matemáticos de forma sistemática como herramientas para trabajar los diferentes contenidos, requiere elaborar indicadores de referencia que permitan evaluar la presencia de los procesos en dichas prácticas. Desde este prisma, construyen y validan la pauta de evaluación "Presencia de los procesos matemáticos en prácticas de enseñanza- aprendizaje de la noción de número entre los 4 y 8 años", que incluye cinco categorías que se corresponden con los cinco procesos indicados por el NCTM (2000), y para cada categoría se aportan siete indicadores de evaluación. De forma más concreta, el diseño, construcción y validación del instrumento contempló seis fases: 1) análisis histórico-epistemológico de los procesos matemáticos y sus significados; 2) estudio de investigaciones sobre los procesos matemáticos en las prácticas docentes del profesorado de Educación Infantil; 3) análisis del tratamiento otorgado a los procesos matemáticos en el currículo; 4) construcción de la versión piloto del instrumento; 5) revisión mediante el juicio de 17 expertos en Didáctica de las Matemáticas de España, Estados Unidos, Chile y Argentina; y 6) construcción de la versión final del instrumento. Las fases 1, 2 y 3 consideran la revisión de literatura e investigaciones que permiten diseñar el instrumento, mientras que las fases 4, 5 y 6 se relacionan específicamente con la construcción y validación del instrumento.

El objetivo de este nuevo estudio es analizar la fiabilidad de su consistencia interna, entendida como la estabilidad en las puntuaciones que 
proporciona si se administrara en repetidas ocasiones al mismo grupo de profesores.

\section{Los procesos matemáticos en las primeras edades}

Las aportaciones contemporáneas relativas a la organización y la construcción del conocimiento matemático subrayan la necesidad de incorporar los procesos matemáticos desde las primeras etapas educativas. Así, por ejemplo, una de las diez recomendaciones de la Asociación Nacional para la Educación Infantil y el Consejo Nacional de Profesores de Matemáticas de Estados Unidos para lograr una educación matemática de alta calidad, enfatiza que los maestros y otros profesionales deberían "utilizar currículos y prácticas docentes que fortalezcan los procesos infantiles de resolución de problemas y razonamiento, así como los de representación, comunicación y conexión de las ideas matemáticas" (NAEYC y NCTM, 2002, p. 5).

Desde esta óptica, se realiza una revisión de las principales aportaciones acerca de los procesos matemáticos en las prácticas de aula.

\section{Resolución de Problemas}

La Asociación de Profesores de Matemáticas de Estados Unidos plantea cuatro objetivos sobre la resolución de problemas (NCTM, 2000): a) construir nuevo conocimiento matemático por medio de la resolución de problemas; b) resolver problemas que surgen de las matemáticas y en otros contextos; c) aplicar y adaptar una variedad de estrategias apropiadas para resolver problemas; y d) controlar y reflexionar sobre el proceso de resolver problemas matemáticos. Partiendo de la base que un problema matemático es diferente que un ejercicio de aplicación (Puig, 1996), debe considerarse que ya desde las primeras edades las situaciones problemáticas no pueden resolverse escuchando al maestro ni repitiendo, sino que se aprende a resolver problemas haciendo, manipulando, simulando, discutiendo, compartiendo, imaginando, observando, visualizando, etc. (Martínez, 2001). De acuerdo con Baroody (1988) deberían plantearse a los niños distintos tipos de situaciones, rutinarias y no rutinarias, con enunciado tanto gráfico como verbal. Alsina (2006) expone una posible secuencia de tipo de problemas a trabajar en las primeras edades: situaciones reales; situaciones dramatizadas; situacio- 
nes manipulativas; una parte del enunciado con material y la otra parte verbal; situaciones gráficas, con imágenes e ilustraciones; enunciado oral-respuesta oral; enunciado oral-respuesta gráfica; enunciado gráficorespuesta gráfica; introducción al enunciado escrito y la respuesta oral o gráfica; introducción al enunciado escrito y la respuesta escrita. Se trata, en definitiva, de partir de lo concreto para avanzar progresivamente a lo simbólico, considerando tres ejes en todo momento: enseñar para, sobre y a través de la resolución de problemas.

\section{RAZONAMIENTO Y PRUEBA}

En relación al razonamiento y la prueba, se plantean cuatro objetivos que deberían trabajarse progresivamente en el aula de matemáticas (NCTM, 2000): a) reconocer el razonamiento y la prueba como aspectos fundamentales de las matemáticas; b) formular e investigar conjeturas matemáticas (por ejemplo formulando a los niños buenas preguntas que les animen a construir nuevo conocimiento sobre lo que ya saben); c) desarrollar y evaluar argumentos y pruebas matemáticas (preguntándoles por ejemplo cómo saben que algo es verdad para que desarrollen progresivamente destrezas que les permitan verificar o refutar sus propias aserciones); y d) escoger y usar varios tipos de razonamiento y métodos de prueba adecuados a su edad. Baroody (1988) expone que en las primeras edades el razonamiento es sobre todo informal y se refiere principalmente a la capacidad de explicar, argumentar o justificar las acciones realizadas y las proposiciones, mientras que la prueba implica comprobar el resultado de dichas acciones y proposiciones, más que demostrarlas o validarlas (la demostración, tal como se entiende en matemáticas, corresponde a etapas posteriores). De acuerdo con Carpenter y Levi (1999), una gestión de las prácticas matemáticas que favorezca el razonamiento y la prueba en las primeras edades implica plantear buenas preguntas, más que dar explicaciones, para que los niños puedan generalizar usando ejemplos y contraejemplos que les permitan comprobar sus generalizaciones. En relación a las buenas preguntas, Sullivan y Lilburn (2002) señalan tres características: a) más que recordar un hecho o reproducir una acción, requieren comprensión de la tarea, aplicación de técnicas y estrategias y análisis y síntesis de los conceptos implicados; b) permiten que los niños aprendan respondiendo, y que los maestros aprendan a partir de las respuestas de los alumnos; y c) permiten diversas respuestas aceptables. 


\section{COMUNICACIÓN}

Desde el punto de vista de la educación matemática, en los últimos años ha aumentado mucho el interés por el estudio de la comunicación y sus repercusiones en el aprendizaje de las matemáticas. Se han analizado varios aspectos, como por ejemplo la diversidad de significados surgidos en las interacciones que se producen en la clase, los elementos que dificultan la construcción de significados compartidos o bien la carencia de negociación (Planas, 2005). Estos estudios ponen de manifiesto que el trabajo de matemáticas en cualquier nivel educativo requiere integrar los procesos de interacción, diálogo y negociación alrededor de los contenidos matemáticos y su gestión, puesto que en la clase de matemáticas los alumnos a menudo interpretan las normas establecidas de maneras diferentes, y muy a menudo también estas interpretaciones difieren de las que los maestros esperan. Nos referimos, sobre todo, al conjunto de significados legitimados que delimitan la cultura del aula, como por ejemplo qué se acepta como situación problemática, a qué criterios se da prioridad en el proceso de resolución de un problema, qué papel se otorga al profesorado, etc. Se trata, pues, de reconocer ya desde las primeras edades la diversidad de significados de las ideas matemáticas y favorecer la participación de todos los alumnos. La carencia de diálogo, sobre todo en los grupos de alumnos de los que se tienen bajas expectativas de éxito, conduce a prácticas matemáticas descontextualizadas y alejadas de la vida cotidiana.

La comunicación es, pues, otro de los procesos matemáticos que debería contemplarse de forma sistemática en las prácticas matemáticas. En este sentido, cabe considerar que el lenguaje oral y el escrito son herramientas imprescindibles, y previas al lenguaje simbólico, para desarrollar y comunicar el pensamiento matemático. Desde este prisma, se deberían considerar cuatro objetivos referentes a la comunicación en el aula de matemáticas (NCTM, 2000): a) organizar y consolidar el pensamiento matemático a través de la comunicación; b) comunicar el pensamiento matemático con coherencia y claridad a los compañeros, maestros y otras personas; c) analizar y evaluar las estrategias y el pensamiento matemático de los demás; y d) usar el lenguaje matemático para expresar ideas matemáticas con precisión. En este sentido, Alsina (2011) señala que los niños empiezan muy pronto a comunicar matemáticamente, por lo que desde que se incorporan en la escuela se les deberían ofrecer oportunidades para interactuar (con otros alumnos, con el maes- 
tro, etc.) y para comunicar las propias ideas matemáticas y escuchar las de los demás.

\section{CONEXIONES}

Respecto a las conexiones matemáticas, se consideran tres objetivos que deberían trabajarse progresivamente en el aula de matemáticas (NCTM, 2000): a) reconocer y usar las conexiones entre ideas matemáticas; b) comprender cómo las ideas matemáticas se interconectan y construyen una sobre otras para producir un todo coherente; y c) reconocer y aplicar las matemáticas en contextos no matemáticos. Desde esta óptica, Alsina (2011) distingue dos tipos de conexiones: las conexiones intradisciplinares, que se refieren a las relaciones entre los diferentes bloques de contenido matemático y entre los contenidos y los procesos matemáticos; y las conexiones interdisciplinares, que incluyen las relaciones de las matemáticas con otras áreas de conocimiento bien con el entorno. En el caso de las etapas de Educación Infantil y Primaria, hace ya varios años que en los currículos -tanto a nivel internacional como español- se insiste en plantear el trabajo de los alumnos para que se favorezcan estas conexiones, sobre todo a partir del momento en el que se deja atrás un currículo orientado a la adquisición de contenidos para avanzar hacia un currículo orientado a la adquisición de competencias. Muchos autores abogan también por este enfoque: por ejemplo, el trabajo de las matemáticas a partir de cuentos y canciones (Saá, 2002), a partir del arte (Edo, 2008), o bien a partir de la psicomotricidad (Benavides y Núñez, 2007), entre otras disciplinas.

\section{REPRESENTACIÓN}

Finalmente, en la literatura acerca de los procesos matemáticos se ha considerado también la representación de las ideas y procedimientos matemáticos a través de objetos físicos, el lenguaje natural, gestos, dibujos, diagramas y símbolos inventados o convencionales, aunque éstos últimos en menor medida. Se plantean tres objetivos referentes a la representación que se deberían trabajar progresivamente en el aula de matemáticas (NCTM, 2000): a) crear y usar representaciones para organizar, registrar, y comunicar ideas matemáticas; b) seleccionar, aplicar y traducir representaciones matemáticas para resolver problemas; y c) usar representaciones por modelizar e interpretar fenómenos físicos, sociales y matemáticos. Se indica que la representación de las ideas y procedi- 
mientos matemáticos es un proceso indispensable para poder aprender. Si no hay representación no hay comprensión, y sin comprensión no puede haber aprendizaje de las matemáticas, pero debe considerarse que el desarrollo progresivo de la representación de las ideas y procedimientos matemáticos va de lo concreto a lo abstracto. En este sentido, Alsina (2011), indica que en el aula debería favorecerse el desarrollo y el uso de múltiples representaciones, analizar las representaciones de los alumnos y modelizar formas convencionales de representación para promover que los alumnos comprendan que la representación es una herramienta para modelizar e interpretar fenómenos de naturaleza matemática que se encuentran en contextos diferentes. Romero y Castro (2008) sugieren que el marco idóneo para trabajar las representaciones y los modelos matemáticos son actividades que sean verdaderas situaciones problemáticas, como por ejemplo los proyectos globalizados, dado que un contexto de aprendizaje atractivo por los alumnos es mucho más valioso que los ejercicios formales o los problemas rutinarios.

Con base en estos antecedentes se ha elaborado el cuestionario para analizar la presencia de los procesos matemáticos en prácticas de enseñanza-aprendizaje de las matemáticas (Coronata, 2014). El objetivo de este trabajo, como se ha indicado, es analizar la fiabilidad interna de dicho instrumento.

\section{Material y métodos}

El cuestionario usado en este estudio está compuesto por los 35 ítems originales de la pauta de evaluación "Presencia de los procesos matemáticos en prácticas de enseñanza- aprendizaje de la noción de número entre los 4 y 8 años" (Coronata, 2014), que representan 35 indicadores agrupados de 7 en 7 , en 5 grupos que se corresponden con los cinco procesos del NCTM (2000): resolución de problemas, razonamiento y prueba, conexiones, comunicación y representación. Cada ítem del cuestionario se ha medido en una escala Likert 5 (Anexo 1).

Veinte evaluadores recogieron información sobre la práctica docente de 102 maestros, de un total de 20 colegios de Educación Infantil y Primaria ( 3 a 5 años y 6 a 12 años de edad respectivamente), puesto que el cuestionario se diseñó originalmente para alumnos de edades comprendidas en estas dos etapas educativas. Los evaluadores fueron formados 
para poder evaluar la aparición o puesta en práctica de los procesos en el aula en el contexto de la asignatura "Innovación e Investigación Matemática en Educación Infantil" del Máster de Innovación e Investigación en Educación Infantil y Primaria de la Universidad de Murcia.

La recogida de datos se efectuó por medio de entrevistas y observación directa de la práctica docente, y se contrastaron con los conocimientos previos que ya tenían los evaluadores acerca de las prácticas de los profesores entrevistados, el 69\%(68) habían sido tutores de prácticas. Las entrevistas seguían siempre el mismo formato: en primer lugar se recogían datos descriptivos (fecha de la entrevista, nombre de la escuela, nivel educativo, años de profesión, género y tutorización del prácticum); y en la segunda parte de la entrevista, el entrevistador leía los ítems del cuestionario y el entrevistado indicaba un valor de la escala Likert 5 en función de su propia percepción sobre la presencia de cada uno de los indicadores del cuestionario en su práctica docente. De łos 102 maestros entrevistados se eliminaron 7 entrevistas por no estar completas, resultando así un total de 95 entrevistas a maestros, por lo tanto 95 cuestionarios cumplimentados.

Los datos se han analizado con el paquete estadístico de R Core Team (2014) R versión 3.1.0 sobre una plataforma i686-pc-linux-gnu (32-bit). Hemos empleado el paquete psych (Revelle, 2015) para el cálculo del coeficiente alfa de Cronbach.

\subsection{Análisis de la fiabilidad}

Descripción de la muestra. Se cuenta con 95 observaciones o mediciones para cada ítem, entre maestros y maestras que fundamentalmente ejercían en el nivel de infantil (47.37\%) como de primaria (52.63\%). El $87.4 \%$ (83) de los cuestionarios correspondió a maestras mientras que el $12.6 \%$ (12) a maestros. De estos 12 maestros solo 2 ejercían en infantil. Las maestras mostraron un reparto más uniforme entre los dos niveles educativos: $51.8 \%$ (43) en infantil y $48.2 \%$ (40) primaria. En cuanto a experiencia laboral no se encontraron diferencias por sexo siendo la media de años trabajados para el conjunto de encuestados: 15.32 con una desviación típica de 10.44..

Alfa de Cronbach

Se ha analizado si los ítems agrupados en cada "proceso" correlacio- 
nan entre sí a fin de construir un instrumento válido de medición de la aparición de cada proceso. Con tal fin empleamos el método de consistencia interna basado en el alfa de Cronbach que permite estimar la fiabilidad de un instrumento de medida compuesto por un conjunto de ítems, medidos en escalas Likert 5, que esperamos midan la misma dimensión teórica (el mismo constructo). De esta manera son sumables en una puntuación única que mide un rasgo que es importante en la construcción teórica del instrumento. La fiabilidad de la escala debe obtenerse siempre con los datos de cada muestra para garantizar la medida fiable del constructo en la muestra concreta de investigación. Los alfas de Cronbach obtenidas para cada proceso (constructo) las mostramos en la Tabla 1 para cada etapa educativa: infantil y primaria.

Tabla 1. Alfas de Cronbach estandarizadas para los procesos en infantil y primaria.

\begin{tabular}{cccc}
\hline $\mathbf{n}^{\mathbf{0}}$ & Proceso matemático & Infantil & Primaria \\
\hline 1 & Resolución de problemas & .81 & .90 \\
2 & Razonamiento y prueba & .87 & .89 \\
3 & Conexiones & .79 & .81 \\
4 & Comunicación & .78 & .83 \\
5 & Representación & .74 & .87 \\
\hline
\end{tabular}

En la Tabla 2 se exponen los alfas para cada proceso sin diferenciar la etapa educativa:

Tabla 2. Alfas de Cronbach estandarizadas para los procesos sin distinguir entre etapa educativa.

\begin{tabular}{ccc}
\hline $\mathbf{n}^{\mathbf{0}}$ & Proceso matemático & alfa-std \\
\hline 1 & Resolución de problemas & .86 \\
2 & Razonamiento y prueba & .88 \\
3 & Conexiones & .82 \\
4 & Comunicación & .81 \\
5 & Representación & .79 \\
\hline
\end{tabular}

El coeficiente alfa de Cronbach, basado en puntuaciones estandarizadas, más bajo obtenido corresponde al quinto proceso "Representación", con un valor de .74 que, según el criterio de George y Mallery (2006), se puede valorar como una consistencia interna aceptable. En 
general, siguiendo los parámetros de estos mismos autores, la valoración media de la consistencia interna es buena (coeficiente alfa medio en Educación Infantil .798 y en Educación Primaria .86). Estos valores de los coeficientes de alfa de Cronbach obtenidos permiten afirmar que todos los ítems están relacionados significativamente con aquellos construidos para evaluar la misma faceta del factor, por lo que forman parte del mismo constructo.

\section{Consideraciones finales}

Los resultados de este estudio aportan datos para poder evaluar de forma fiable la presencia de los procesos matemáticos en la práctica docente del profesorado. En concreto, se ha obtenido un coeficiente alfa de Cronbach medio de .832 que, según el criterio de George y Mallery (2006), se puede valorar como una buena consistencia interna. El coeficiente alfa más alto se ha obtenido en "Resolución de Problemas" en Educación Primaria, con un valor de .90, la resolución de problemas ha tenido tradicionalmente una presencia importante en el currículo de matemáticas de Educación Primaria, ya sea como contenido a enseñar o como herramienta para construir conocimiento matemático, en la línea planteada por Puig (1996). El coeficiente alfa más bajo se ha obtenido en "Representación" en Educación Infantil, con un valor de .74. En cambio, el resto de procesos no han tenido la misma consideración en las orientaciones curriculares. En particular, la representación es un proceso poco mencionado en la etapa de Educación Infantil, ya que suele asociarse a lo simbólico y en esta etapa la mayoría de aprendizajes se realizan desde lo concreto, esto puede ser un motivo para el coeficiente obtenido.

En general, pues, según los coeficientes obtenidos, el cuestionario descrito constituye una herramienta base para medir la presencia o ausencia de los procesos matemáticos en la práctica docente, además de orientar al profesorado para poderlos incorporar en las prácticas de aula.

La incorporación de los procesos matemáticos en la práctica docente es todavía deficitaria, y todavía lo es más su evaluación a partir de instrumentos válidos y fiables, debido a varios factores como por ejemplo la poca investigación en educación matemática en las primeras edades hasta hace pocos años, la escasa formación inicial sobre estos aspectos 
o bien la falta de una presencia explícita de los procesos matemáticos en los currículos hasta hace pocos años (Alsina, 2009, 2012, 2013). Según este autor, en el caso español se ha producido un punto de inflexión en las orientaciones curriculares vigentes (BOE, 2007, 2014) al mencionar las formas de trabajar los contenidos a través de distintos procesos como la resolución de problemas, la comunicación y la representación, etc. Asimismo, la revisión de algunos programas de Didáctica de las Matemáticas como consecuencia, principalmente, de la incorporación en el Espacio Europeo de Educación Superior ha permitido también dar mayor énfasis a los procesos de pensamiento matemático. Y, por último, otro factor que puede haber contribuido a enfatizar la importancia de los procesos matemáticos en las prácticas de enseñanza es, principalmente, la divulgación de los resultados de la investigación en educación matemática infantil que se realiza en España, fruto de de la creación del Grupo de Investigación en Educación Matemática Infantil (IEMI) de la Sociedad Española de Investigación en Educación Matemática (SEIEM) en el año 2011. A pesar de este cambio de tendencia, que no es todavía generalizado, la aportación de un cuestionario válido y fiable para medir la presencia de los procesos matemáticos en las prácticas de aula puede contribuir no solo a evaluar este aspecto, sino también a ofrecer orientaciones sobre qué tipos de prácticas docentes son necesarias para aumentar dicha presencia.

En síntesis, pues, este trabajo otorga información que puede ser de interés para el profesorado en activo, los futuros profesores y los formadores de profesores en torno a la incorporación y evaluación de los procesos matemáticos en la práctica docente del profesorado de las primeras edades, sobre todo considerando el papel fundamental de estos procesos en el desarrollo de la competencia matemática de los alumnos. Consideramos, sin embargo, que este cuestionario es un factor de partida de un proceso formativo que debe contribuir, tanto en el marco de la formación inicial como permanente del profesorado, a considerar de forma explícita los procesos matemáticos para la planificación y evaluación de actividades matemáticas competenciales en el aula (de Guzman, 2001; Niss, 2002; Alsina, 2011, 2015; entre otros). Por esta razón, en futuros trabajos será necesario diseñar programas de formación específicos que, con base en estos resultados, permitan mejorar el grado de presencia de los procesos matemáticos en la práctica docente. 
Los procesos matemáticos en la práctica docente: análisis de la fiabilidad de un cuestionario de evaluación

antonio Maurandi López, Ángel Alsina y Claudia Coronata Segure

\section{Referencias}

Alsina, Á. (2006). ¿Cómo desarrollar el pensamiento matemático de 0 a 6 años? Barcelona: Octaedro-Eumo.

Alsina, Á. (2009). Un análisis optimista de la educación matemática en la formación de maestros de educación infantil. UNO, Revista de Didáctica de las Matemáticas, 51, 30-43.

Alsina, Á. (2011). Aprendre a usar les matemàtiques. Els processos matemàtics: propostes didàctiques per a l'Educació Infantil. Vic: Eumo Editorial.

Alsina, Á. (2012). Más allá de los contenidos, los procesos matemáticos en Educación Infantil. Edma 0-6: Educación Matemática en la Infancia, 1(1), 1-14.

Alsina, Á. (2013). Early Childhood Mathematics Education: Research, Currículum, and Educational Practice. Journal of Research in Mathematics Education, 2(1), 100-153.

Alsina, Á. (2015). Cómo fomentar el aprendizaje de las matemáticas en el aula. Ideas clave para la Educación Primaria. Barcelona: Editorial Casals.

Alsina, Á. y Coronota, C. (2014). Los procesos matemáticos en las prácticas docentes: diseño, construcción y validación de un instrumento de evaluación. Edma 0-6: Educación Matemática en la Infancia, 3(2), 21-34.

Baroody, A.J. (1988). El pensamiento matemático de los niños. Madrid: Visor, MEC.

Benavides, M. y Núnez, R. (2007). Matemática y psicomotricidad: la noción de espacio. Revista Iberoamericana de Psicomotricidad y Técnicas Corporales, 25, 7(1), 235-244.

BOE (2007). Orden ECl/3960/2007, de 19 de diciembre, por la que se establece el currículo y se regula la ordenación de la educación infantil. Recuperado de: http://www. boe.es/boe/dias/2008/01/05/pdfs/A01016-01036.pdf

BOE (2014). Real Decreto 126/2014, de 28 de febrero, por el que se establece el currículo básico de la Educación Primaria. BOE (2014), n 52, 14, 19349-19420.

Carpenter, T.P. y Levi, L. (1999). Developing conceptions of algebraic reasoning in the primary grades. Madison: University of Wisconsin-Madison.

Coronata, C. (2014). Presencia de los procesos matemáticos en la enseñanza del número de 4 a 8 años. Transición entre la Educación Infantil y Elemental. Tesis doctoral. Girona: Universidad de Girona. Recuperado de: http://dugi-doc.udg.edu/handle/10256/9750

De Guzmán, M. (2001). Tendencias actuales de la educación matemática. Sigma, 19, 5-25.

Edo, M. (2008). Matemáticas y arte en educación infantil. UNO. Revista de Didáctica de las Matemáticas, 47, 37-53.

George, D. y Mallery, P. (2006). Spss for Windows step by step: A Simple Guide and Reference. 13.0 Update ( $6^{\mathrm{a}}$ ed.). Boston: Pearson Education, Inc.

Llinares, S. (2008). Agendas de investigación en Educación Matemática en España. Una aproximación desde "ISI-web of knowledge" y ERIH. En R. Luengo, B.Gómez, M. Camacho y L. Blanco (Eds), Investigación en educación Matemática XII (pp. 25-54). Badajoz: SEIEM.

Martínez, C. (2001). La resolució de problemes en les primeres edats. Biaix, 18, 9-12.

NAEYC y NCTM (2002). Early childhood mathematics: Promoting good beginnings. A 
Los procesos matemáticos en la práctica docente: análisis de la fiabilidad de un cuestionario de evaluación Antonio Maurandi López, Ángel Alsina y Claudia Coronata Segure

joint position statement. Recuperado de: http://www.naeyc.org/files/naeyc/file/positions/psmath.pdf

NCTM (2000). Principles and standards for school mathematics. Reston, Va.: The National Council of Teachers of Mathematics

Niss, M. (2002). Mathematical competencies and the learning of mathematics: the Danish Kom Project. Roskilde: Roskilde University.

OECD (2004). Learning for tomorrow's world: First results from PISA 2003. Paris: OECD.

OECD (2006). Assessing scientific, reading and mathematical literacy: A framework from PISA 2006. París: OECD.

OECD (2007). PISA 2006 Science competence for tomorrow's world. Paris: OECD.

Planas, N. (2005). El papel del discurso en la construcción del Discurso de la práctica matemática. Cultura y Educación, 17(1), 19-34.

Puig, L. (1996). Elementos de resolución de problemas. Granada: Comares.

R Core Team (2014). R: A language and environment for statistical computing. Viena: $R$ Foundation for Statistical Computing. Recuperado de: http://www.R-project.org.

Revelle, W. (2015). Psych: Procedures for Personality and Psychological Research. Evanston, Illinois: Northwestern University. Recuperado de: http://CRAN.R-project.org/ package $=$ psych Version $=1.5 .1$.

Romero, S. y Castro, F. (2008). Modelización matemática en secundaria desde un punto de vista superior: EL PROBLEMA DE DOBOGÓKÓ. Modelling in Science Education and Learning, 1(2), 11-23.

Saá, M.D. (2002). Las matemáticas de los cuentos y las canciones. Madrid: Editorial Eos.

Sullivan, P. y Lilburn, P. (2002). Good questions for Math Teaching. Australia: Oxford University Press. 



\section{Anexo 1: Cuestionario}

Tomando en consideración las limitaciones de la formación inicial y permanente en relación a la Didáctica de las Matemáticas, y también la escasa valoración que se otorga a las matemáticas y su enseñanza a nivel social (por ejemplo, los opiniones de los medios de comunicación ante los malos resultados españoles en pruebas internacionales como PISA, TIMSS, etc.), se pretende conocer la opinión del profesorado en activo sobre los aspectos que se deberían contemplar en las prácticas de enseñanza de las matemáticas, por encima de los comentarios adversos de los medios de comunicación.

Con la finalidad de conocer su opinión profesional, le solicitamos que rellene este breve cuestionario anónimo.

\section{Fecha:}

Escuela:

Nivel Educativo en el que ejerce:

Años de profesión:

Género:

¿Ha tutorizado el prácticum del Grado de Maestro del/la estudiante que le administra el cuestionario?

\begin{tabular}{l}
$\mathrm{SI} \quad \mathrm{NO}$ \\
$\begin{array}{l}\text { iHa tutorizado a algún otro alumno/a?: } \\
\mathrm{SI} \quad \mathrm{NO}\end{array}$ \\
\hline
\end{tabular}

Indique con una escala del 1 al 5 el grado de presencia de cada indicador en SU práctica habitual de la enseñanza de las matemáticas (1 nada, 5 mucho): 


\section{Indicadores de RESOLUCIÓN DE PROBLEMAS:}

Plantea situaciones problemáticas usando diferentes tipos de apoyo (oral, concreto, pictórico).

Contextualiza las situaciones problemáticas a la vida cotidiana de los alumnos.

Propone situaciones problemáticas de diversos tipos.

Realiza preguntas que generan la investigación y exploración para solucionar al problema.

Permite a los niños la utilización de material concreto y/o pictórico con apoyo oral para la resolución de problemas.

Mantiene a los niños comprometidos con el proceso de resolución de problemas.

Promueve la discusión en torno a las estrategias de resolución de problemas y los resultados.

\section{Indicadores de RAZONAMIENTO Y PRUEBA: $\quad \begin{array}{lllll}1 & 2 & 3 & 4 & 5\end{array}$}

Invita a hacer conjeturas.

Permite que los propios alumnos descubran, analicen y propongan diversas vías de resolución.

Pide a los alumnos que expliquen, justifiquen o argumenten las estrategias o técnicas que utilizaron durante la resolución.

Plantea interrogantes para que los alumnos argumenten sus respuestas.

Promueve que los alumnos comprueben conjeturas de la vida cotidiana.

Promueve el apoyo del razonamiento matemático.

Entrega retroalimentación con material concreto permitiendo el pensamiento divergente.

\section{Indicadores de CONEXIONES:}

Considera las experiencias matemáticas cotidianas de los alumnos para avanzar hacia las matemáticas más formales.

Realiza conexiones entre diversos contenidos matemáticos.

Desarrolla actividades matemáticas vinculadas a contextos musicales.

Trabaja las matemáticas vinculándolas con la literatura infantil.

Relaciona las matemáticas con la expresión artística.

Genera conocimiento matemático a través de contextos vinculados a la psicomotricidad.

Promueve que los alumnos apliquen el conocimiento matemático a las situaciones de la vida cotidiana. 


\section{Indicadores de COMUNICACIÓN:}

$\begin{array}{lllll}1 & 2 & 3 & 4 & 5\end{array}$

Promueve con mayor énfasis la comunicación en el aula que la entrega de información unidireccional.

Favorece la interacción con otros para aprender y comprender las ideas matemáticas.

Impulsa el intercambio de ideas matemáticas a través del lenguaje oral, gesticular, gráfico, concreto y /o simbólico.

Pide al niño explicitar con lenguaje matemático adecuado sus estrategias y respuestas.

Incentiva en los alumnos el respeto por la forma de pensar y de exponer sus puntos de vista en torno al contenido matemático.

Fomenta la escucha atenta de los puntos de vista de los demás. Interviene mayoritariamente a través de preguntas, más que a través de explicaciones.

\section{Indicadores de REPRESENTACIÓN:}

Pide a los niños que hablen, escuchen y reflexionen sobre las matemáticas para avanzar hacia la representación simbólica.

Utiliza materiales concretos como recursos para representar ideas matemáticas.

Utiliza modelos ejemplificadores (esquemas, entre otros) para mostrar maneras de resolver situaciones problemáticas.

Trabaja en los niños las representaciones concretas (dibujos, etc.).

Trabaja en los niños las representaciones pictóricas (signos, etc.).

Trabaja en los niños las representaciones simbólicas (notación convencional).

Muestra un trabajo bidireccional (de lo concreto a lo abstracto y de lo abstracto a lo concreto). 
